Copyright (C) 2014 IEEE. Personal use of this material is permitted. Permission from IEEE must be obtained for all other uses, in any current or future media, including reprinting/republishing this material for advertising or promotional purposes, creating new collective works, for resale or redistribution to servers or lists, or reuse of any copyrighted component of this work in other works. 


\title{
MSE Based Transceiver Designs for Bi-directional Full-Duplex MIMO Systems
}

\author{
Ali Cagatay Cirik*, Rui Wang ${ }^{\dagger}$, Yue Rong ${ }^{\ddagger}$ and Yingbo Hua* \\ * Department of Electrical Engineering, University of California Riverside, Riverside, CA, 92521 \\ $\dagger$ Department of Information and Communications, Tongji University, Shanghai, 201804, P. R. China \\ $\ddagger$ Department of Electrical and Computer Engineering, Curtin University, Bentley, WA 6102, Australia \\ Email: \{acirik, yhua\}@ee.ucr.edu, liouxingrui@gmail.com, y.rong@curtin.edu.au
}

\begin{abstract}
We consider a multiple antenna full-duplex (FD) bidirectional (point-to-point) communication system with a limited analog domain self-interference cancellation capability. The effect of the residual self-interference resulting from independent and identically distributed (i.i.d.) channel estimation errors and limited dynamic ranges of the transmitters and receivers is studied in the digital domain. We design transceiver matrices based on the minimization of sum mean-squared error (MSE) and the maximum per-node MSE optimization problems subject to individual power constraints at each node through an iterative alternating algorithm, which is proven to converge to at least a local optimal solution.
\end{abstract}

Index Terms-Full-duplex, limited dynamic range, MIMO, MSE, self-interference, transceiver designs.

\section{INTRODUCTION}

Full-duplex bi-directional wireless communication systems, which perform simultaneous transmission and reception at the same time and frequency has the capability to double the system spectral efficiency, compared to that of half-duplex systems, in which the nodes transmit and receive on orthogonal channels. The potential advantages of full-duplex radios over half-duplex radios have recently attracted a great deal of attention and motivated experimental [1]-[5] and theoretical research activities [6]-[12].

The main challenge to implement full-duplex radios is the self-interference signal from the transmitter chain into the receiver chain, which can saturate the front-end of the receiver, and must be canceled. A full-duplex radio design using signal inversion and adaptive cancellation was proposed in [1]. In [2], self-interference is canceled by generating a canceling signal and adding it at the receive antenna using radio-frequency (RF) adder in the analog domain. Analog domain cancellation for a single antenna and multiple antenna full-duplex system was studied in [3], [4] and [5], respectively.

The increased spatial diversity with the use of multiple-input multiple-output (MIMO) systems enables transmit beamforming techniques to mitigate the self-interference [6]-[9]. Nullspace projection and minimum mean-squared error (MMSE) techniques are used in [6] to mitigate the self-interference in the spatial domain, which are possible when there are enough degrees of freedom (d.o.f.). An effective techique under insufficient number of d.o.f. is proposed in [7] that maximize the signal-to-interference ratio at the relay input and output. In [8], a self-interference cancellation algorithm based on the optimization of the relay processing vectors over the continuous domain is proposed, which outperforms the techniques in [6]. In [9], transmit and receive beamforming and power allocation techniques for MIMO full-duplex/half-duplex decode-and-forward/amplify-and-forward relays are discussed.

Most of the works on optimization problems related to fullduplex systems have studied the maximization of the achievable rate [10]-[12] and to the best of our knowledge, MSE based transceiver designs have not been studied. MSE is an important performance measure to approach the informationtheoretic limits of Gaussian channels, and has been widely considered as an optimization metric in precoding design in the literature, e.g., [13]-[15]. In this paper, a joint and iterative transceiver design for the MIMO bi-directional full-duplex systems is proposed. We assume that the nodes have imperfect channel state information (CSI) of the MIMO channels, and we consider the limited dynamic ranges of the transmitters and receivers. Minimization of both sum-MSE and the maximum per-node MSE subject to individual power constraints at each node is studied, and an alternating iterative algorithm to compute the optimum transmit and receiving matrices is proposed, which is guaranteed to converge to at least a local optimal solution.

The notations used in this paper are as follows. $(\cdot)^{T}$ and $(\cdot)^{H}$ are the transpose and conjugate transpose, respectively. $\mathrm{E}\{\cdot\}$ denotes the statistical expectation; $\mathbf{I}_{N}$ is the $N$ by $N$ identity matrix; $\operatorname{tr}(\cdot)$ and are the trace and determinant of a matrix; $\operatorname{diag}(\mathbf{A})$ is the diagonal matrix with the elements on the diagonal of $\mathbf{A}$. vec $(\cdot)$ is a one long column vector obtained by stacking the elements of a matrix. $\otimes, \perp$ and $\|\cdot\|_{2}$ denote Kronecker product, the statistical independence, and Euclidean norm of a vector, respectively. We will also refer to full-duplex as FD and half-duplex as HD.

\section{SySTEM MODEL}

We consider a FD MIMO system between two nodes exchanging information simultaneously as seen in Fig. 1. We assume, without loss of generality, that each node has $N$ physical antennas that can transmit and receive simultaneously at the same carrier frequency [5].

As illustrated in Fig. 1, since the nodes operate in FD mode, the receiver $i \in\{1,2\}$ receives signals from both transmitters over MIMO channels, the entries of which are assumed to i.i.d. 


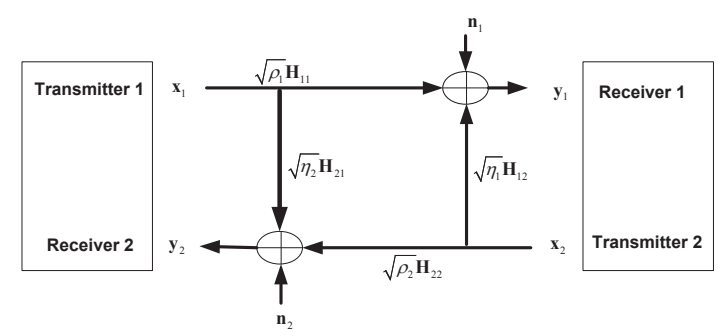

Fig. 1. Bi-directional Full-Duplex MIMO System

complex Gaussian variables with zero mean and unit variance. $\mathbf{H}_{i i} \in \mathbb{C}^{N \times N}$ is the channel between $i$ th transmitter-receiver pair and $\mathbf{H}_{i j} \in \mathbb{C}^{N \times N}, j \in\{1,2\}$ and $j \neq i$ is the selfinterference channel from the transmitter $j$ to the receiver $i{ }^{1}$ The channel matrices are assumed to be constant during a time slot, but change randomly at each time slot.

We assume that both nodes estimate the channels, and because of the channel estimation errors, they have imperfect CSI. We use the channel estimation model in [6], [7], where the receiver $i \in\{1,2\}$ applies the MMSE estimation method to estimate $\mathbf{H}_{i j}$. The MMSE estimation and the estimation error are denoted as $\tilde{\mathbf{H}}_{i j}$ and $\Delta \mathbf{H}_{i j}=\mathbf{H}_{i j}-\tilde{\mathbf{H}}_{i j}$, respectively. Here $\tilde{\mathbf{H}}_{i j}$ and $\Delta \mathbf{H}_{i j}$ are uncorrelated, and the entries of $\Delta \mathbf{H}_{i j}$ are complex Gaussian with zero mean and variance $\sigma_{e}^{2}$.

The data streams at the $i$ th transmitter are denoted as $\mathbf{d}_{i} \in$ $\mathbb{C}^{N}, i=1,2$, which are assumed to be complex, zero mean, i.i.d with $\mathrm{E}\left\{\mathbf{d}_{i} \mathbf{d}_{i}^{H}\right\}=\mathbf{I}_{N}$, and $\mathrm{E}\left\{\mathbf{d}_{i} \mathbf{d}_{j}^{H}\right\}=\mathbf{0}_{N}, i \neq j$.

The transmitted signal by the $i$ th transmitter of size $N \times 1$ is given by

$$
\mathbf{x}_{i}=\mathbf{V}_{i} \mathbf{d}_{i}, \quad i=1,2
$$

where $\mathbf{V}_{i} \in \mathbb{C}^{N \times N}$ is the precoding matrix at the $i$ th transmitter, and $\mathbf{x}_{i}$ is assumed to be Gaussian distributed with zero mean and covariance matrix $\mathrm{E}\left\{\mathbf{x}_{i} \mathbf{x}_{i}^{H}\right\}=\mathbf{V}_{i} \mathbf{V}_{i}^{H}$.

We consider a FD MIMO bi-directional system, where two nodes transmit simultaneously. Thus, the receiver $i$ receives the transmission signals from both transmitters. The received signal at the $i$ th receiver of size $N \times 1$ is written as

$$
\begin{aligned}
\mathbf{y}_{i}= & \sqrt{\rho}_{i} \mathbf{H}_{i i}\left(\mathbf{x}_{i}+\mathbf{c}_{i}\right)+\sqrt{\eta} \mathbf{H}_{i j}\left(\mathbf{x}_{j}+\mathbf{c}_{j}\right)+\mathbf{e}_{i}+\mathbf{n}_{i} \\
= & \sqrt{\rho}_{i} \tilde{\mathbf{H}}_{i i} \mathbf{x}_{i}+\sqrt{\rho}_{i} \Delta \mathbf{H}_{i i} \mathbf{x}_{i}+\sqrt{\rho}_{i} \mathbf{H}_{i i} \mathbf{c}_{i}+\sqrt{\eta}_{i} \tilde{\mathbf{H}}_{i j} \mathbf{x}_{j} \\
& +\sqrt{\eta}_{i} \Delta \mathbf{H}_{i j} \mathbf{x}_{j}+\sqrt{\eta}_{i} \mathbf{H}_{i j} \mathbf{c}_{j}+\mathbf{e}_{i}+\mathbf{n}_{i}, i=1,2
\end{aligned}
$$

where $\mathbf{x}_{j}=\mathbf{V}_{j} \mathbf{d}_{j}$ is $N \times 1$ the self-interference signal at the $i$ th receiver, which is Gaussian distributed with zero mean and covariance matrix $\mathrm{E}\left\{\mathbf{x}_{j} \mathbf{x}_{j}^{H}\right\}=\mathbf{V}_{j} \mathbf{V}_{j}^{H}$. Note that, the number of streams is assumed to be equal to the number of antennas, $N . \mathbf{n}_{i} \in \mathbb{C}^{N}$ is the additive white Gaussian noise (AWGN) vector at the $i$ th receiver with zero mean and unit covariance matrix, $\mathrm{E}\left\{\mathbf{n}_{i} \mathbf{n}_{i}^{H}\right\}=\mathbf{I}_{N}$ and it is uncorrelated to both $\mathbf{x}_{i}$ and

\footnotetext{
${ }^{1}$ Before analog domain cancellation, the self-interference channel has a strong Line-Of-Sight (LOS) component, so can be modeled as Ricean distribution with a large $K$-factor. After applying an analog domain cancellation, the strong LOS component is attenuated, resulting in a Ricean distribution with a small $K$-factor or a Rayleigh distribution (under large suppression) [2].
}

$\mathbf{x}_{j} . \rho_{i}$ denotes the average power gain of the $i$ th transmitterreceiver link, and $\eta_{i}$ denotes the average power gain of the self-interference channel.

$\mathbf{c}_{k} \in \mathbb{C}^{N}, k=1,2$ is the transmitter distortion injected at each transmitter antenna of the $k$ th transmitter that models the effect of limited dynamic range (DR) by approximating the effects of power-amplifier noise, non-linearities in the DAC and phase noise [10]. The covariance matrix of $\mathbf{c}_{k}$ is given by $\kappa(\kappa \ll 1)$ times the energy of the intended signal at each transmit antenna. In particular $\mathbf{c}_{k}$ can be modeled as [10]

$$
\mathbf{c}_{k} \sim \mathcal{C N}\left(\mathbf{0}, \kappa \operatorname{diag}\left(\mathbf{V}_{k} \mathbf{V}_{k}^{H}\right)\right), \quad \mathbf{c}_{k} \perp \mathbf{x}_{k} .
$$

$\mathbf{e}_{k} \in \mathbb{C}^{N}, k=1,2$ is the receiver distortion injected at each receive antenna of the $k$ th receiver that models the effect of limited receiver DR by approximating the effects of additive gain-control noise, non-linearities in the ADC and phase noise [10]. The covariance matrix of $\mathbf{e}_{k}$ is given by $\beta(\beta \ll 1)$ times the energy of the undistorted received signal at each receive antenna. In particular, $\mathbf{e}_{k}$ can be modeled as [10]

$$
\mathbf{e}_{k} \sim \mathcal{C N}\left(\mathbf{0}, \beta \operatorname{diag}\left(\boldsymbol{\Phi}_{k}\right)\right), \quad \mathbf{e}_{k} \perp \mathbf{u}_{k}
$$

where $\boldsymbol{\Phi}_{k}=\operatorname{Cov}\left\{\mathbf{u}_{k}\right\}$ and $\mathbf{u}_{k}$ is the $k$ th receiver's undistorted received vector, i.e. $\mathbf{u}_{k}=\mathbf{y}_{k}-\mathbf{e}_{k}$.

Since the receiver $i \in\{1,2\}$ has the knowledge of the selfinterference signal $\mathbf{x}_{j}$ from transmitter $j \in\{1,2\}, j \neq i$, the self-interference term $\sqrt{\eta}_{i} \tilde{\mathbf{H}}_{i j} \mathbf{x}_{j}$ can be canceled [10], and the resulting received signal can be written as

$$
\begin{aligned}
\tilde{\mathbf{y}}_{i} & =\mathbf{y}_{i}-\sqrt{\eta} \tilde{\mathbf{H}}_{i j} \mathbf{x}_{j} \\
& =\sqrt{\rho_{i}} \tilde{\mathbf{H}}_{i i} \mathbf{x}_{i}+\mathbf{v}_{i}
\end{aligned}
$$

where $\mathbf{v}_{i}$ is the total noise after the self-interference cancellation due to channel estimation errors and limited DR of the transmitters/receivers, and is given by

$$
\begin{aligned}
\mathbf{v}_{i}= & \sqrt{\rho_{i}} \Delta \mathbf{H}_{i i} \mathbf{x}_{i}+\sqrt{\rho}_{i} \mathbf{H}_{i i} \mathbf{c}_{i}+\sqrt{\eta} \Delta \mathbf{H}_{i j} \mathbf{x}_{j} \\
& +\sqrt{\eta} \mathbf{H}_{i j} \mathbf{c}_{j}+\mathbf{e}_{i}+\mathbf{n}_{i} .
\end{aligned}
$$

Similar to the analysis in [10], under $\kappa \ll 1$ and $\beta \ll 1$, the covariance matrix of $\mathbf{v}_{i}$ can be approximated as

$$
\begin{aligned}
\tilde{\boldsymbol{\Sigma}}_{i} \approx & \rho_{i} \kappa \tilde{\mathbf{H}}_{i i} \operatorname{diag}\left(\mathbf{V}_{i} \mathbf{V}_{i}^{H}\right) \tilde{\mathbf{H}}_{i i}^{H}+\rho_{i} \sigma_{e}^{2} \operatorname{tr}\left\{\mathbf{V}_{i} \mathbf{V}_{i}^{H}\right\} \mathbf{I}_{N} \\
& +\eta_{i} \kappa \tilde{\mathbf{H}}_{i j} \operatorname{diag}\left(\mathbf{V}_{j} \mathbf{V}_{j}^{H}\right) \tilde{\mathbf{H}}_{i j}^{H}+\eta_{i} \sigma_{e}^{2} \operatorname{tr}\left\{\mathbf{V}_{j} \mathbf{V}_{j}^{H}\right\} \mathbf{I}_{N} \\
& +\beta \rho_{i} \operatorname{diag}\left(\tilde{\mathbf{H}}_{i i} \mathbf{V}_{i} \mathbf{V}_{i}^{H} \tilde{\mathbf{H}}_{i i}^{H}\right)+\mathbf{I}_{N} \\
& +\beta \eta_{i} \operatorname{diag}\left(\tilde{\mathbf{H}}_{i j} \mathbf{V}_{j} \mathbf{V}_{j}^{H} \tilde{\mathbf{H}}_{i j}^{H}\right), i, j \in\{1,2\} \text { and } j \neq i .
\end{aligned}
$$

To estimate the data streams, node $i$ applies the linear receiver $\mathbf{R}_{i}, i=1,2$.

$$
\begin{aligned}
\hat{\mathbf{d}}_{i} & =\mathbf{R}_{i} \tilde{\mathbf{y}}_{i} \\
& =\sqrt{\rho}{ }_{i} \mathbf{R}_{i} \tilde{\mathbf{H}}_{i i} \mathbf{V}_{i} \mathbf{d}_{i}+\mathbf{R}_{i} \mathbf{v}_{i} .
\end{aligned}
$$


Using (8), the MSE matrix at the $i$ th receiver can be expressed as

$$
\begin{aligned}
\mathbf{M S E}_{i}= & \mathrm{E}\left\{\left(\hat{\mathbf{d}}_{i}-\mathbf{d}_{i}\right)\left(\hat{\mathbf{d}}_{i}-\mathbf{d}_{i}\right)^{H}\right\} \\
= & \left(\sqrt{\rho} \mathbf{R}_{i} \tilde{\mathbf{H}}_{i i} \mathbf{V}_{i}-\mathbf{I}_{N}\right)\left(\sqrt{\rho} \mathbf{R}_{i} \tilde{\mathbf{H}}_{i i} \mathbf{V}_{i}-\mathbf{I}_{N}\right)^{H} \\
& +\mathbf{R}_{i} \tilde{\mathbf{\Sigma}}_{i} \mathbf{R}_{i}^{H}
\end{aligned}
$$

\section{SUM-MSE MiNIMIZATION}

Sum-MSE optimization problem can formulated as

$$
\begin{array}{cl}
\min _{\mathbf{V}_{i}, \mathbf{R}_{i}} & \sum_{i=1}^{2} \operatorname{tr}\left\{\mathbf{M S E}_{i}\right\} \\
\text { s.t } & \operatorname{tr}\left\{\mathbf{V}_{i} \mathbf{V}_{i}^{H}\right\} \leq P_{i}, \quad i=1,2
\end{array}
$$

where $P_{i}$ is the individual power constraint at the $i$ th transmitter.

Note that the sum-MSE optimization problem (10)-(11) is not jointly convex over transmit precoding matrices $\mathbf{V}_{i}$ and receiving filter matrices $\mathbf{R}_{i}$, so the standard convex optimization methods can not be applied. But since sum-MSE optimization problem (10)-(11) is component-wise convex over $\mathbf{V}_{i}$ and $\mathbf{R}_{i}$, an iterative alternating algorithm that finds the efficient solution of $\mathbf{V}_{i}, \mathbf{R}_{i}, i=1,2$ based on the necessary conditions of the optimization problem (10)-(11) is carried out.

The Lagrange function of the sum-MSE problem (10)-(11) is written as:

$\mathcal{L}\left(\mathbf{V}_{i}, \mathbf{R}_{i}, \lambda_{i}\right)=\sum_{i=1}^{2} \operatorname{tr}\left\{\mathbf{M S E}_{i}\right\}+\sum_{i=1}^{2} \lambda_{i}\left(\operatorname{tr}\left\{\mathbf{V}_{i} \mathbf{V}_{i}^{H}\right\}-P_{i}\right)$

where $\lambda_{i}$ is the Lagrange multiplier associated with the individual power constraint at the transmitter $i$. The KarushKuhn-Tucker (KKT) conditions can be written as

$$
\begin{aligned}
& \operatorname{tr}\left\{\mathbf{V}_{i} \mathbf{V}_{i}^{H}\right\}-P_{i} \leq 0, \quad i=1,2 \\
& \lambda_{i} \geq 0, \quad i=1,2 \\
& \lambda_{i}\left(\operatorname{tr}\left\{\mathbf{V}_{i} \mathbf{V}_{i}^{H}\right\}-P_{i}\right)=0, \quad i=1,2 \\
& \frac{\partial \mathcal{L}}{\partial \mathbf{V}_{i}^{*}}=\mathbf{0}, \quad \frac{\partial L}{\partial \mathbf{R}_{i}^{*}}=\mathbf{0}, \quad i=1,2 .
\end{aligned}
$$

Taking the partial derivative of the Lagrangian function $\mathcal{L}$ with respect to the matrix $\mathbf{V}_{i}$ and $\mathbf{R}_{i}$, we can obtain

$$
\begin{aligned}
\frac{\partial \mathcal{L}}{\partial \mathbf{V}_{i}^{*}} & =\lambda_{i} \mathbf{V}_{i}-\sqrt{\rho_{i}} \tilde{\mathbf{H}}_{i i}^{H} \mathbf{R}_{i}^{H}+\mathbf{X}_{i} \mathbf{V}_{i} \\
\frac{\partial \mathcal{L}}{\partial \mathbf{R}_{i}^{*}} & =-\sqrt{\rho_{i}} \mathbf{V}_{i}^{H} \tilde{\mathbf{H}}_{i i}^{H}+\rho_{i} \mathbf{R}_{i} \tilde{\mathbf{H}}_{i i} \mathbf{V}_{i} \mathbf{V}_{i}^{H} \tilde{\mathbf{H}}_{i i}^{H}+\mathbf{R}_{i} \tilde{\boldsymbol{\Sigma}}_{i}
\end{aligned}
$$

where $\mathbf{X}_{i}$ in (16) is given by

$$
\begin{aligned}
\mathbf{X}_{i}= & \rho_{i} \tilde{\mathbf{H}}_{i i}^{H} \mathbf{R}_{i}^{H} \mathbf{R}_{i} \tilde{\mathbf{H}}_{i i}+\rho_{i} \kappa \operatorname{diag}\left(\tilde{\mathbf{H}}_{i i}^{H} \mathbf{R}_{i}^{H} \mathbf{R}_{i} \tilde{\mathbf{H}}_{i i}\right) \\
& +\rho_{i} \sigma_{e}^{2} \operatorname{tr}\left\{\mathbf{R}_{i} \mathbf{R}_{i}^{H}\right\} \mathbf{I}_{N}+\rho_{i} \beta \tilde{\mathbf{H}}_{i i}^{H} \operatorname{diag}\left(\mathbf{R}_{i}^{H} \mathbf{R}_{i}\right) \tilde{\mathbf{H}}_{i i} \\
& +\eta_{j} \kappa \operatorname{diag}\left(\tilde{\mathbf{H}}_{j i}^{H} \mathbf{R}_{j}^{H} \mathbf{R}_{j} \tilde{\mathbf{H}}_{j i}\right)+\eta_{j} \sigma_{e}^{2} \operatorname{tr}\left\{\mathbf{R}_{j} \mathbf{R}_{j}^{H}\right\} \mathbf{I}_{N} \\
& +\eta_{j} \beta \tilde{\mathbf{H}}_{j i}^{H} \operatorname{diag}\left(\mathbf{R}_{j}^{H} \mathbf{R}_{j}\right) \tilde{\mathbf{H}}_{j i}, i=1,2 .
\end{aligned}
$$

From (16)-(17), the optimal $\mathbf{V}_{i}$ and $\mathbf{R}_{i}, i=1,2$ can be expressed as

$$
\begin{aligned}
\mathbf{V}_{i} & =\sqrt{\rho_{i}}\left(\lambda_{i} \mathbf{I}_{N}+\mathbf{X}_{i}\right)^{-1} \tilde{\mathbf{H}}_{i i}^{H} \mathbf{R}_{i}^{H} \\
\mathbf{R}_{i} & =\sqrt{\rho_{i}} \mathbf{V}_{i}^{H} \tilde{\mathbf{H}}_{i i}^{H}\left(\rho_{i} \tilde{\mathbf{H}}_{i i} \mathbf{V}_{i} \mathbf{V}_{i}^{H} \tilde{\mathbf{H}}_{i i}^{H}+\tilde{\boldsymbol{\Sigma}}_{i}\right)^{-1}
\end{aligned}
$$

As it is seen from (19)-(20) that the optimal transmit precoding matrix $\mathbf{V}_{i}$ is dependent on the receiving filter matrices of two nodes, $\mathbf{R}_{i}, i=\{1,2\}$ and the optimal receiving matrix $\mathbf{R}_{i}$, which is a linear MMSE receiver, is dependent on the optimal transmit precoding matrices of two nodes, $\mathbf{V}_{i}, i=\{1,2\}$. Therefore, we obtain the transmit precoding and receive filtering matrices using an iterative alternating technique. Particularly, we compute and update the optimal transmit matrices $\mathbf{V}_{i}$ from (19) when the receive filters $\mathbf{R}_{i}, i=\{1,2\}$ are fixed, and then using the transmit matrices $\mathbf{V}_{i}$ obtained at the previous step, we compute and update the receiver filter matrices $\mathbf{R}_{i}$ from (20). The iterations are repeated until convergence or a pre-defined number of iterations is reached.

The values of the Lagrange multipliers $\lambda_{i}, i=1,2$ are calculated by taking the singular value decomposition of $\mathbf{X}_{i}^{[n+1]}=\mathbf{U}_{i}^{[n+1]} \boldsymbol{\Delta}_{i}^{[n+1]}\left(\mathbf{U}_{i}^{[n+1]}\right)^{H}$ and writing the update as $\mathbf{V}_{i}^{\left[\tilde{\lambda}_{i}\right]}=\sqrt{\rho_{i}}\left(\tilde{\lambda}_{i} \mathbf{I}_{N}+\mathbf{X}_{i}^{[n+1]}\right)^{-1} \tilde{\mathbf{H}}_{i i}^{H}\left(\mathbf{R}_{i}^{[n+1]}\right)^{H}$ at each iteration. By plugging $\mathbf{V}_{i}^{\left[\tilde{\lambda_{i}}\right]}$ into the power constraint in (11) and after simple steps, (11) can be written as

$$
\operatorname{tr}\left\{\mathbf{V}_{i}^{\left[\tilde{\lambda_{i}}\right]}\left(\mathbf{V}_{i}^{\left[\tilde{\lambda_{i}}\right]}\right)^{H}\right\}=\rho_{i} \sum_{k=1}^{N} \frac{g_{i k}^{[n+1]}}{\left(\tilde{\lambda_{i}}+\Delta_{i k}^{[n+1]}\right)^{2}}=P_{i}
$$

where $g_{i k}^{[n+1]}$ denotes the $k$ th element of $\left(\mathbf{U}_{i}^{[n+1]}\right)^{H} \tilde{\mathbf{H}}_{i i}^{H}$ $\left(\mathbf{R}_{i}^{[n+1]}\right)^{H} \mathbf{R}_{i}^{[n+1]} \tilde{\mathbf{H}}_{i i} \mathbf{U}_{i}^{[n+1]}$ and $\Delta_{i k}^{[n+1]}$ denotes the $k$ th element of the matrix $\boldsymbol{\Delta}_{i}^{[n+1]}$. We can compute $\tilde{\lambda}_{i}, i=1,2$ from (21) numerically using Bisection search. The values of the Lagrange multipliers $\lambda_{i}, i=1,2$ are equal to $\tilde{\lambda}_{i}, i=1,2$ if $\tilde{\lambda}_{i}, \quad i=1,2$ is non-negative. Otherwise, the Lagrange multipliers $\lambda_{i}, i=1,2$ are assigned as zeros.

Since the proposed iterative alternating algorithm decreases the sum-MSE monotonically at each iteration, and the fact that MSE is bounded below (at least by zero), it is easily seen that the proposed algorithm is convergent and guaranteed to converge to at least a local optimal solution.

\section{Min-Max MSE Minimization}

In the sum-MSE transceiver design problem discussed in Section III, each node can have different MSEs, which may not be fair. On the other hand, the Min-Max per-node MSE transceiver design problem ensures that each receiver has the same MSE so that it introduces fairness among the two FD nodes. The Min-Max MSE optimization problem is formulated as:

$$
\begin{aligned}
\min _{\mathbf{V}_{i}, \mathbf{R}_{i}} \max _{i=1,2} & \operatorname{tr}\left\{\mathbf{M S E}_{i}\right\} \\
\text { s.t } & \operatorname{tr}\left\{\mathbf{V}_{i} \mathbf{V}_{i}^{H}\right\} \leq P_{i}, \quad i=1,2 .
\end{aligned}
$$


Similar to the sum-MSE optimization problem (10)-(11), the Min-Max MSE optimization problem is not jointly convex over transmit precoding matrices $\mathbf{V}_{i}$ and receive filtering matrices $\mathbf{R}_{i}$. Therefore we again apply the iterative alternating algorithm to compute the optimal $\mathbf{V}_{i}$ or $\mathbf{R}_{i}$ while keeping the other one fixed. For fixed receiver filtering matrices $\mathbf{R}_{i}, i=1,2$, the Min-Max optimization problem to compute the optimal transmit precoding matrix can be written as:

$$
\begin{aligned}
\min _{\mathbf{V}_{i}} \max _{i=1,2} & \operatorname{tr}\left\{\mathbf{M S E} \mathbf{E}_{i}\right\} \\
\text { s.t } & \operatorname{tr}\left\{\mathbf{V}_{i} \mathbf{V}_{i}^{H}\right\} \leq P_{i}, \quad i=1,2 .
\end{aligned}
$$

By introducing an auxiliary variable $l$, which is an upper bound on the square root of $\operatorname{tr}\left\{\mathbf{M S E}_{i}\right\}$ (i.e., $\left.\sqrt{\operatorname{tr}\{\mathbf{M S E}}\right\} \leq l$ ), the optimization problem (24)-(25) can be rewritten as

$$
\begin{array}{cl}
\min _{\mathbf{V}_{i}, l} & l \\
\text { s.t } & \sqrt{\operatorname{tr}\left\{\mathbf{M S E}_{i}\right\}} \leq l \quad i=1,2 \\
& \operatorname{tr}\left\{\mathbf{V}_{i} \mathbf{V}_{i}^{H}\right\} \leq P_{i}, \quad i=1,2 .
\end{array}
$$

To solve the optimization problem in (26)-(28), we need to write $\operatorname{tr}\left\{\mathbf{M S E}_{i}\right\}$ in vector form. As shown in Appendix, $\operatorname{tr}\left\{\mathbf{M S E}_{i}\right\}$ can be written as

$$
\begin{aligned}
& \operatorname{tr}\left\{\mathbf{M S E}_{i}\right\} \\
& \begin{array}{c}
{\left[\mathbf{I}_{N} \otimes\left(\sqrt{\rho_{i}} \mathbf{R}_{i} \tilde{\mathbf{H}}_{i i}\right)\right] \operatorname{vec}\left(\mathbf{V}_{i}\right)-\operatorname{vec}\left(\mathbf{I}_{N}\right)} \\
\sqrt{\rho_{i} \kappa}\left[\mathbf{I}_{N} \otimes\left(\left(\operatorname{diag}\left(\tilde{\mathbf{H}}_{i i}^{H} \mathbf{R}_{i}^{H} \mathbf{R}_{i} \tilde{\mathbf{H}}_{i i}\right)\right)^{1 / 2}\right)\right] \operatorname{vec}\left(\mathbf{V}_{i}\right) \\
\sqrt{\rho_{i} \sigma_{e}^{2}} \sqrt{\operatorname{tr}\left\{\mathbf{R}_{i} \mathbf{R}_{i}^{H}\right\}} \operatorname{vec}\left(\mathbf{V}_{i}\right) \\
\sqrt{\beta \rho_{i}}\left[\mathbf{I}_{N} \otimes\left(\left(\operatorname{diag}\left(\mathbf{R}_{i}^{H} \mathbf{R}_{i}\right)\right)^{1 / 2} \tilde{\mathbf{H}}_{i i}\right)\right] \operatorname{vec}\left(\mathbf{V}_{i}\right) \\
\sqrt{\eta_{i} \kappa}\left[\mathbf{I}_{N} \otimes\left(\left(\operatorname{diag}\left(\tilde{\mathbf{H}}_{i j}^{H} \mathbf{R}_{i}^{H} \mathbf{R}_{i} \tilde{\mathbf{H}}_{i j}\right)\right)^{1 / 2}\right)\right] \operatorname{vec}\left(\mathbf{V}_{j}\right) \\
\sqrt{\eta_{i} \sigma_{e}^{2}} \sqrt{\operatorname{tr}\left\{\mathbf{R}_{i} \mathbf{R}_{i}^{H}\right\}} \operatorname{vec}\left(\mathbf{V}_{j}\right) \\
\sqrt{\beta \eta_{i}}\left[\mathbf { I } _ { N } \otimes \left(\left(\operatorname{diag}\left(\mathbf{R}_{i}^{H} \mathbf{R}_{i}\right)\right)^{1 / 2}\right.\right. \\
\left.\sqrt{\operatorname{tr}\left\{\mathbf{R}_{i j} \mathbf{R}_{i}^{H}\right\}}\right] \operatorname{vec}\left(\mathbf{V}_{j}\right)
\end{array} \|_{2}
\end{aligned}
$$$$
\triangleq\left\|\boldsymbol{\mu}_{i}\right\|_{2}^{2} \text {. }
$$

After obtaining the vector form of $\operatorname{tr}\left\{\mathbf{M S E}_{i}\right\}$, the MinMax optimization problem to compute the transmit precoding matrices (26)-(28) can be written as

$$
\begin{array}{cl}
\min _{\mathbf{V}_{i}, l} & l \\
\text { s.t } & \left\|\boldsymbol{\mu}_{i}\right\|_{2} \leq l, \quad i=1,2 \\
& \left\|\operatorname{vec}\left(\mathbf{V}_{i}\right)\right\|_{2} \leq \sqrt{P_{i}}, \quad i=1,2 .
\end{array}
$$

Since the objective function (30) is linear and the constraints (31)-(32) are second-order cones, we can conclude that (30)-(32) is a second-order cone programming (SOCP) problem [16] and can be efficiently solved by standard SOCP solvers.

Under the fixed transmit precoding matrices $\mathbf{V}_{i}$, it is shown in (20) that the optimal $\mathbf{R}_{i}, i=1,2$ is linear MMSE receiver. So overall, the iterative alternating Min-Max MSE algorithm applies linear MMSE receiver, under fixed $\mathbf{V}_{i}$ to obtain the optimal receive filtering matrices $\mathbf{R}_{i}$, and solves (30)-(32), under fixed $\mathbf{R}_{i}$ to obtain the optimal transmit precoding matrices $\mathbf{V}_{i}$. Similar to the discussion on the convergence of the sum-MSE algorithm in Section III, we can also argue that Min-Max MSE algorithm is guaranteed to converge to at least a local optimal solution.

\section{Simulation Results}

We simulate the sum-MSE (denoted as "Total") and MinMax MSE (denoted as "MinMax") transceiver design problems for MIMO FD bi-directional system as a function of channel estimation errors $\sigma_{e}^{2}$ and dynamic range parameters $\kappa$ and $\beta$. For simplicity, we assumed a symmetrical scenario with $\rho_{1}=\rho_{2}=\rho$ and $\eta_{1}=\eta_{2}=\eta$, and the same transmit power constraint at each transmitter, i.e., $P_{i}=N, i=1,2$. Thus, the signal-to-noise ratio (SNR) for the desired channels is defined as $\mathrm{SNR}_{i}=\mathrm{SNR}=\rho N, i=1,2$ and the interference-to-noise ratio (INR) for the self-interference channels $\mathrm{INR}_{i}=\mathrm{INR}=\eta N, i=1,2$. The stopping criteria (the difference between MSE values of the two iterations) and the maximum number of iterations of the proposed iterative alternating algorithm is set to be $10^{-4}$ and 1000 , respectively. The results are averaged over 1000 independent channel realizations. As mentioned before, the sum-MSE and Min-Max MSE optimization problems are non-convex, so initialization point is important to achieve a suboptimal solution with a good performance. In [15], several choices for initial point such as right singular matrices and random matrices initialization have been discussed. In this paper, we use right singular matrices initialization.

In our first example, the effect of channel estimation errors on the MSE of Total and MinMax algorithms is examined. It can be seen from Fig. 2 that as the channel estimation errors increases, the MSE of both Total and MinMax algorithms increases. Also note that channel estimation error produces an irreducible error floor, i.e., MSE can not be further reduced by increasing SNR, since the noise dominates the channel estimation error at low SNR, but at high SNR, the channel estimation error dominates the noise, and the performance is governed by channel estimation error, not noise [17].

In our second example, the MSE of the Total and MinMax algorithms under limited transmitter and receiver DR is compared. It can be seen from Fig. 3 that as $\kappa$ and $\beta$ decrease, the MSE value also decreases and exhibits an error floor. Also, the MSE curves almost overlap at low $\kappa$ and $\beta$ values.

The last example computes the MSE values for each node in the system for the Total and MinMax schemes out of one channel realization. We can see in Fig. 4 that the Total scheme achieves the minimum sum MSE over two nodes and the MinMax scheme introduces fairness, by ensuring that the two nodes have almost the same MSE.

\section{CONCLUSION}

In this work, the effects of residual self-interference, due to the imperfect CSI and limited DR at the transmitters and 


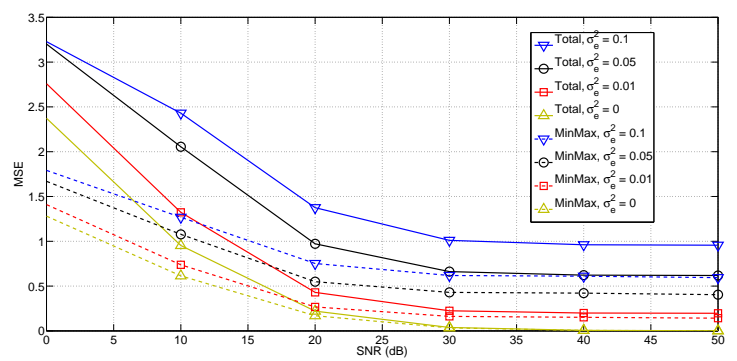

Fig. 2. MSE comparison of the Total and MinMax algorithms with different channel estimation errors versus SNR. Here INR $=20 \mathrm{~dB}, N=2, \kappa=$ $\beta=-40 \mathrm{~dB}$.

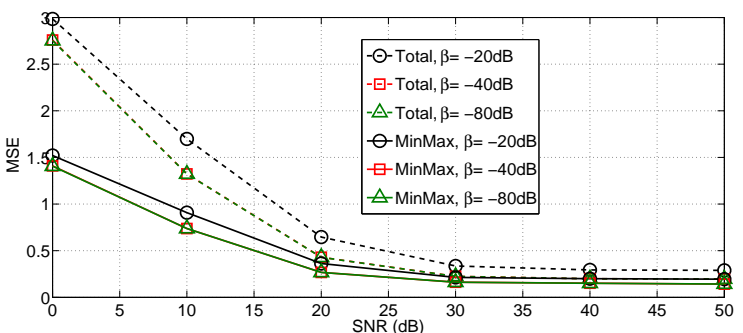

Fig. 3. MSE comparison of the Total and MinMax algorithms with different $\kappa=\beta$ values versus SNR. Here $N=2, \mathrm{INR}=20 \mathrm{~dB}, \sigma_{e}^{2}=0.01$.

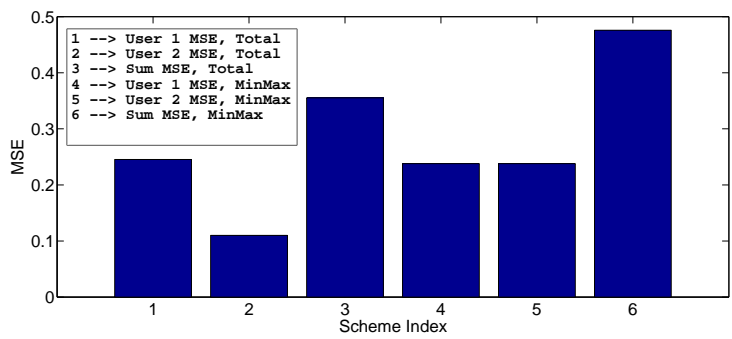

Fig. 4. MSE distribution of Total and MinMax algorithms. The schemes $1-3$ correspond to Total and the schemes 4-6 correspond to MinMax algorithms. For each algorithm, the first two bars are the achieved user MSEs and the third bar is the sum-MSE. Here $N=3, \sigma_{e}^{2}=0.01, \kappa=\beta=-40 \mathrm{~dB}$, $\mathrm{SNR}=20 \mathrm{~dB}$ and $\mathrm{INR}=10 \mathrm{~dB}$.

receivers, on the sum-MSE and Min-Max MSE transceiver design problems for FD MIMO bi-directional system is studied. Since the transceiver design problems are non-convex, an iterative alternating algorithm is proposed that compute the transmit precoding or receive filtering matrices in an alternating fashion while keeping the other one fixed. It is shown through numerical simulations that MSE at each node increases as the channel estimation error and the power of the transmitter/receiver impairments increases. Moreover, in Min-Max MSE transceiver design the nodes achieve the same MSE, which is fair, and sum-MSE transceiver design achieves the minimum total MSE over two FD nodes.

\section{APPENDIX}

After taking the trace of (9) and using $\|\operatorname{vec}(\mathbf{A})\|_{2}^{2}=$ $\operatorname{tr}\left\{\mathbf{A A}^{H}\right\}$, the $\mathrm{MSE}_{i}=\operatorname{tr}\left\{\mathbf{M S E} \mathbf{E}_{i}\right\}$ in (27) can be rewritten as

$$
\begin{aligned}
\operatorname{MSE}_{i}= & \left\|\operatorname{vec}\left(\sqrt{\rho_{i}} \mathbf{R}_{i} \tilde{\mathbf{H}}_{i i} \mathbf{V}_{i}\right)-\operatorname{vec}\left(\mathbf{I}_{N}\right)\right\|_{2}^{2} \\
& +\rho_{i} \kappa\left\|\operatorname{vec}\left(\left(\operatorname{diag}\left(\tilde{\mathbf{H}}_{i i}^{H} \mathbf{R}_{i}^{H} \mathbf{R}_{i} \tilde{\mathbf{H}}_{i i}\right)\right)^{1 / 2} \mathbf{V}_{i}\right)\right\|_{2}^{2} \\
& +\rho_{i} \sigma_{e}^{2} \operatorname{tr}\left\{\mathbf{R}_{i} \mathbf{R}_{i}^{H}\right\}\left\|\operatorname{vec}\left(\mathbf{V}_{i}\right)\right\|_{2}^{2} \\
& +\rho_{i} \beta\left\|\operatorname{vec}\left(\left(\operatorname{diag}\left(\mathbf{R}_{i}^{H} \mathbf{R}_{i}\right)\right)^{1 / 2} \tilde{\mathbf{H}}_{i i} \mathbf{V}_{i}\right)\right\|_{2}^{2} \\
& +\eta_{i} \kappa\left\|\operatorname{vec}\left(\left(\operatorname{diag}\left(\tilde{\mathbf{H}}_{i j}^{H} \mathbf{R}_{i}^{H} \mathbf{R}_{i} \tilde{\mathbf{H}}_{i j}\right)\right)^{1 / 2} \mathbf{V}_{j}\right)\right\|_{2}^{2} \\
& +\eta_{i} \sigma_{e}^{2} \operatorname{tr}\left\{\mathbf{R}_{i} \mathbf{R}_{i}^{H}\right\}\left\|\operatorname{vec}\left(\mathbf{V}_{j}\right)\right\|_{2}^{2}+\operatorname{tr}\left\{\mathbf{R}_{i} \mathbf{R}_{i}^{H}\right\} \\
& +\eta_{i} \beta\left\|\operatorname{vec}\left(\left(\operatorname{diag}\left(\mathbf{R}_{i}^{H} \mathbf{R}_{i}\right)\right)^{1 / 2} \tilde{\mathbf{H}}_{i j} \mathbf{V}_{j}\right)\right\|_{2}^{2} . \quad(33)
\end{aligned}
$$

Using the identity $\operatorname{vec}(\mathbf{A B C})=\left(\mathbf{C}^{T} \otimes \mathbf{A}\right)$ vec $(\mathbf{B})$, (33) can be written as (29).

\section{REFERENCES}

[1] M. Jain, J. I. Choi, T. Kim, D. Bharadia, K. Srinivasan, S. Seth, P. Levis, S. Katti, and P. Sinha, "Practical, real-time, full duplex wireless," in Proc. Mobicom 2011.

[2] M. Duarte, C. Dick, and A. Sabharwal, "Experiment-driven characterization of full-duplex wireless systems," IEEE Trans. on Wireless Communications, vol. 11, no. 12, pp. 4296-4307, Dec. 2012.

[3] M. Knox, "Single antenna full duplex communications using a common carrier," in 13th Annual IEEE Wireless and Microwave Technology Conference (WAMICON), pp. 1-6, April 2012.

[4] Y. Hua, P. Liang, Y. Ma, A. Cirik and Q. Gao, "A method for broadband full-duplex MIMO radio," IEEE Signal Processing Letters, vol. 19, no. 12, pp. 793-796, Dec. 2012.

[5] D. Bharadia and S. Katti, "Full duplex MIMO radios," USENIX NSDI, 2014.

[6] T. Riihonen, S. Werner, and R. Wichman, "Mitigation of loopback selfinterference in full-duplex MIMO relays," IEEE Trans. Signal Process. vol. 59, no. 12, pp. 5983-5993, Dec. 2011.

[7] P. Lioliou, M. Viberg, M. Coldrey, and F. Athley, "Self-interference suppression in full-duplex MIMO relays," in Proc. Asilomar Conf. Signals Syst. Comput., (Pacific Grove, CA), pp. 658-662, Oct. 2010.

[8] B. Chun and H. Park; , "A spatial-domain joint-nulling method of selfinterference in full-duplex relays," IEEE Communications Letters, vol. 16, no. 4, pp. 436-438, April 2012.

[9] Y. Hua, "An overview of beamforming and power allocation for MIMO relays," in Proc. IEEE Military Commun. Conf., (San Jose, CA), pp. 375380, Nov. 2010.

[10] B. P. Day, A. R. Margetts, D. W. Bliss and P. Schniter, "Full-duplex bidirectional MIMO: Achievable rates under limited dynamic range,' IEEE Trans. Signal Process., vol. 60, no. 7, pp. 3702-3713, July 2012.

[11] A. C. Cirik, Y. Rong, and Y. Hua, "Ergodic mutual information of fullduplex MIMO radios with residual self-interference," in Proc. Asilomar Conf. Signals, Systems and Computers, Nov. 2013.

[12] A. C. Cirik, R. Wang, and Y. Hua, "Weighted-sum-rate maximization for bi-directional full-duplex MIMO systems," in Proc. Asilomar Conf. Signals, Systems and Computers, Nov. 2013.

[13] R. Mo and Y. Chew, "MMSE-based joint source and relay precoding design for amplify-and-forward MIMO relay networks," IEEE Trans. Wireless Commun., vol. 8, no. 9, pp. 4668-4676, Sept. 2009.

[14] W. Guan and H. Luo, "Joint MMSE transceiver design in nonregenerative MIMO relay systems," IEEE Commun. Lett., vol. 12, no. 7, pp. 517-519, July 2008.

[15] H. Shen, B. Li, M. Tao, and X. Wang, "MSE-based transceiver designs for the MIMO interference channel," IEEE Trans. Wireless Commun., vol. 9, no. 11, pp. 3480-3489, Nov. 2010.

[16] M. S. Lobo, L. Vandenberghe, S. Boyd, and H. Lebret, "Applications of second order cone programming," Linear Algebra APP., 1998.

[17] T. Yoo and A. J. Goldsmith, "Capacity and power allocation for fading MIMO channels with channel estimation error," IEEE Trans. Inf. Theory, vol. 52, no. 5, pp. 2203-2214, May 2006. 